\title{
An Auto-Landing Solution for a Drop Test RLV Demonstrator
}

\author{
Peng Yong-Tao ${ }^{1}$, Wang Yue-Ping ${ }^{1}$, Wei Wen-Ling ${ }^{1} \&$ Wang Xiao-Ting ${ }^{1}$ \\ ${ }^{1}$ Flight Automatic Control Research Institute, Xi'an, Shanxi, China \\ Correspondence: Wang Xiao-Ting, Flight Automatic Control Research Institute, Xi'an, Shanxi, China. Tel: \\ 86-159-0295-6193. E-mail: lopermanster@gmail.com
}

Received: July 2, $2014 \quad$ Accepted: Juny 7, $2014 \quad$ Online Published: September 9, 2014

doi:10.5539/mas.v8n5p204 URL: http://dx.doi.org/10.5539/mas.v8n5p204

\begin{abstract}
Unpowered drop test is very important for reusable launch vehicle (RLV) autolanding technology development. One of the challenges is to design an autolanding trajectory with enough robustness against uncertainties of drop conditions, aerodynamic characteristic and disturbances from control system and environment. In this paper, a solution including trajectory generation and control design is proposed for a drop test RLV demonstrator. Firstly, the drop test and vertical flight trajectory are introduced. Also, parts of the drop flight, segments of landing trajecory and trajectory design parameters in groups are shown. Secondly, an online trajectory generation method including self-adapted capture segment plan and landing trajectory optimization following UAV auto-landing experience are illustrated in detail by designing groups of parameters. Then, simple but practical gain schedule control laws are presented. Finally, mathematic simulation and analysis based on both RSS and Monte Carlo methods indicate that the solution proposed has shown an acceptable robustness and can provide enough capability for the demonstrator to land saftly.
\end{abstract}

Keywords: Reusable Launch Vehicle (RLV), auto-landing, on-line trajectory plan, RSS analysis, Mento Carlo Analysis

\section{Introduction}

Unpowered drop test is always used for validating the auto-landing ability of a newly developed RLV. The vehicle will be dropped away from the carrier about 7-8 km high above the ground, and slide along an arranged trajectory until landing on the pointed runway. Without propulsion system on board, energy control of such a low lift-to-drag vehicle is very difficult. That is why the vertical auto-landing trajectory should be carefully designed before the test, to ensure the safety and enhance the robustness and reliability.

Shuttle unpowered auto-landing trajectory design method (Tsikalas, 1982) is worth learning because of the successful development and applications of Shuttle. The nominal trajectory comprises four segments: steep glideslope, circular flare, exponential flare and shallow glideslope. During the steep glideslope segment, energy and trajectory dispersions caused by TAEM flight will be removed by establishing and stabilizing expected velocity. The circular flare employs a circular arc to linearly increase the flight path angle from steep to shallow. The exponential flare is necessary for avoiding acceleration discontinuity during the transition onto the shallow glideslope. And in the shallow glideslope, the vehicle flies with a relatively small vertical velocity until the landing flare sets the craft on the runway.

With the same segment settings, an Auto-landing I-load Program (ALIP) is developed for X-34 (Barton and Tragesser, 1999), which enforces physical constraints such as loads, vertical descent rate, continuity, and smoothness reduces the design problem to a two-point boundary value problem(TBVP) with conditions on the initial and final dynamic pressure. The ALIP is able to decrease design time and add substantial robustness to off nominal conditions.

In this paper, a developed auto-landing solution for an unpowered drop test RLV demonstrator is proposed, including a drop test trajectory design method and classic control laws. In the second and third section, an on-line trajectory generation method based on the ALIP is illustrated, which is improved for better safeties in these two aspects: 1) to increase robustness of guidance against drop conditions, a self-adapted capture segment is added in front of the steep glideslope segment; 2) to ensure the touchdown performance, the position and dynamic pressure of the final plan point of TBVP, which is set to be the starting point of the shallow gideslope segment, is determined according to UAV auto-landing trajectory design experience. In the forth section, classic 
gain scheduling control laws which are simple but focus on engineering are introduced. In the last section, the Root Sum Square (RSS) Method and Monte Carlo Method are used for simulation analysis and evaluation of the proposed solution in multi-disturbance circumstances.

\section{Vertical Flight Trajectory Design}

The design of vertical auto-landing trajectory of a drop RLV demonstrator is a two-point boundary value problem, with the initial and ending states specified. The design would process the velocity profile depending on the height profile, in order to meet the constrained state of touchdown. With the vehicle pointing directly to the runway, the whole flight comprises 4 parts: free flight, attitude stabilizing, capturing, and landing. The landing segment also comprises 6 segments: Steep Glideslope, Circular Flare, Exponential Decay, Shallow Glideslope, Landing Flare and runway running, as shown in Fig. 1.

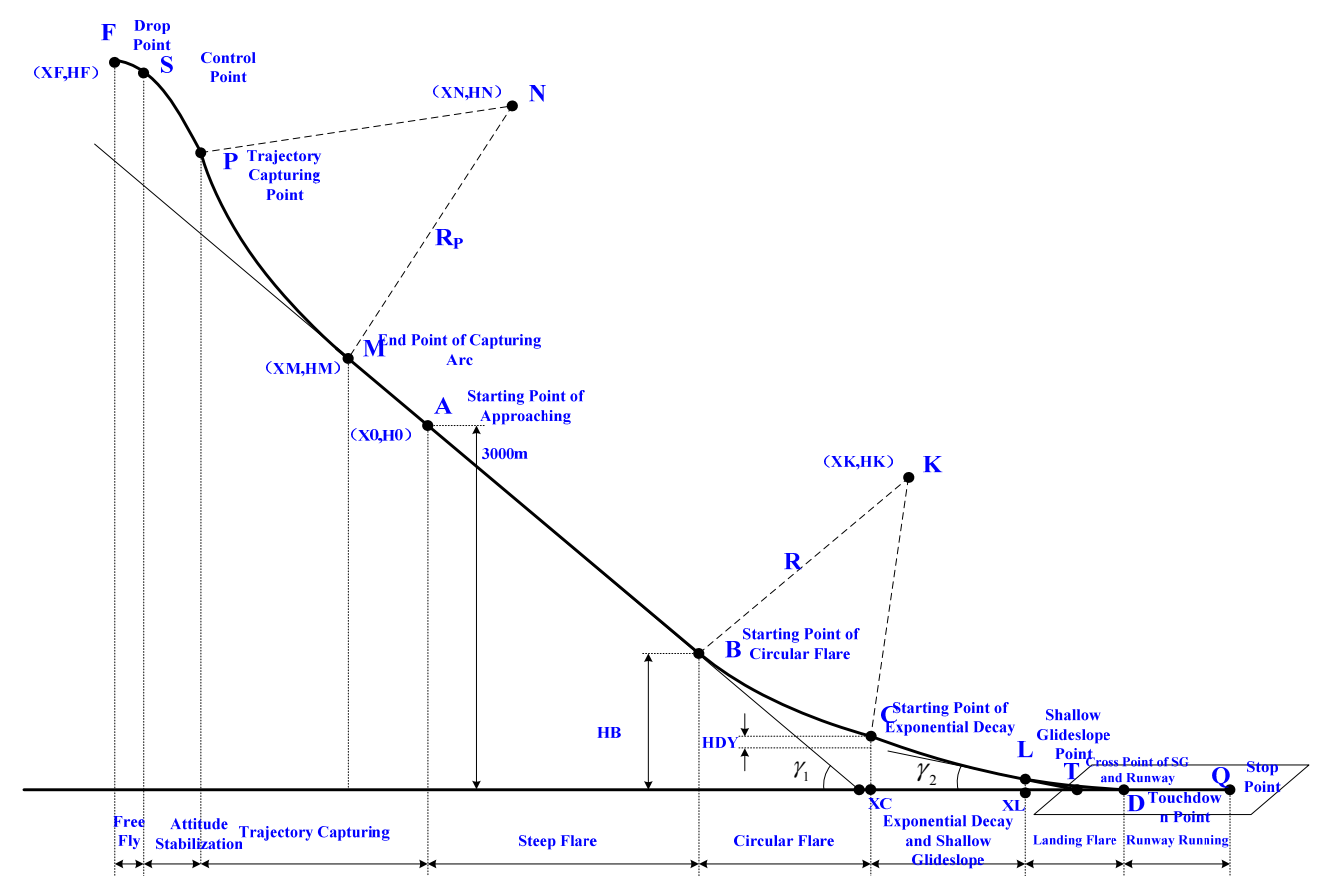

Figure 1. Vertical flight trajectory of drop test with the demonstrator pointing toward the runway

$>$ During the Free Flight Segment, the vehicle will not have any control input, in order to prevent the interference between the carrier aircraft and the demonstrator, ensuring the safety of carrier aircraft;

$>$ During the attitude stabilization segment, the attitude of the demonstrator will be controlled to a prescribed value;

$>$ During the Capturing segment, a trajectory would be generated according to the current position of the demonstrator, the height and horizontal displacement would be controlled, with the reduce of the initial position error;

$>$ During the Steep Glideslope Segment, a trajectory following will be performed, stabilizing the demonstrator flight in the glideslope;

$>$ The segments of Circular Flare and Exponential Decay will control the demonstrator from a large path angle to a smaller one;

$>$ Vertical velocity control will be performed at the final Shallow Glideslope segment, to satisfy the constrained descending rate of touchdown.

Considering the flight states in each segment, the segments contain the need of trajectory design starting from the Capturing Segment, and ending at the Exponential Decay segment. The mathematical representation of the autolanding trajectory is in ground axes, with the prescribed touchdown point $\mathrm{D}$ as the origin, using the direction pointing to the farther end of runway as $\mathrm{X}$, and the lateral direction to the right size of the runway as $\mathrm{Z}$, up as $\mathrm{Y}$. The relationship between vertical altitude (h) of the demonstrator and the downrange distance, $\mathrm{X}$, can be 
expressed as the following equations.

Circular part of Trajectory Capturing:

$$
H=H_{N}-\sqrt{R_{P}{ }^{2}-\left(X-X_{N}\right)^{2}} \quad X<X_{M}
$$

Landing Steep glideslope:

$$
H=H_{A}+\tan \gamma_{1}\left(X-X_{A}\right) \quad X<X_{A}
$$

Steep glideslope:

$$
H=H_{B}+\tan \gamma_{1}\left(X_{B}-X\right) \quad X<X_{B}
$$

Circular flare:

$$
H=H_{K}-\sqrt{R^{2}-\left(X-X_{K}\right)^{2}} \quad X<X_{C}
$$

Exponential Decay and shallow glideslope:

$$
H=X \cdot \tan \gamma_{2}+H_{D Y} \cdot e^{\left(X_{C}-X\right) / \sigma} \quad X<X_{L}
$$

The description of parameters in the equations is presented in Tab. 1, the first 10 parameters define the trajectory geometry of landing segments, and parameters 11-14 define the trajectory of capturing segment. The last parameter indicates the drop point of the demonstrator.

Table 1. Trajectory design parameters

\begin{tabular}{lll}
\hline Symbol & Unit & Description \\
\hline$\gamma_{1}$ & rad & Steep glideslope \\
$\gamma_{2}$ & rad & Shallow glideslope \\
$\mathrm{L}\left(\mathrm{X}_{\mathrm{L}}, \mathrm{H}_{\mathrm{L}}\right)$ & $\mathrm{m}$ & $\begin{array}{l}\text { Horizontal coordinate of starting point of Shallow Glideslope segment } \\
\mathrm{R}\end{array}$ \\
$\mathrm{K}\left(\mathrm{X}_{\mathrm{K}}, \mathrm{H}_{\mathrm{K}}\right)$ & $\mathrm{m}$ & Radius of the arc \\
$\mathrm{C}\left(\mathrm{X}_{\mathrm{C}}, \mathrm{H}_{\mathrm{C}}\right)$ & $\mathrm{m}$ & The coordinate of origin $\mathrm{K}$ \\
$\mathrm{H}_{\mathrm{DY}}$ & $\mathrm{m}$ & The coordinate of starting point of Exponential Segment \\
$\mathrm{B}\left(\mathrm{X}_{\mathrm{B}}, \mathrm{H}_{\mathrm{B}}\right)$ & $\mathrm{m}$ & Cooportion Factor of Exponential Flare \\
$\sigma$ & $\mathrm{m}$ & Decay rate of the Exponential Flare \\
$\mathrm{A}\left(\mathrm{X}_{\mathrm{A}}, \mathrm{H}_{\mathrm{A}}\right)$ & $\mathrm{m}$ & Coordinate of the touchdown point \\
$\mathrm{R}_{\mathrm{P}}$ & $\mathrm{m}$ & The radius of the arc of Capturing Segment \\
$\mathrm{N}\left(\mathrm{X}_{\mathrm{N}}, \mathrm{H}_{\mathrm{N}}\right)$ & $\mathrm{m}$ & The coordinate of origin of the arc of Capturing Segment \\
$\mathrm{M}\left(\mathrm{X}_{\mathrm{M}}, \mathrm{H}_{\mathrm{M}}\right)$ & $\mathrm{m}$ & The coordinate of the finishing point of arc trajectory \\
$\mathrm{P}\left(\mathrm{X}_{\mathrm{P}}, \mathrm{H}_{\mathrm{P}}\right)$ & $\mathrm{m}$ & The coordinate of the starting point of Capture Segment \\
$\mathrm{F}\left(\mathrm{X}_{\mathrm{F}}, \mathrm{H}_{\mathrm{F}}\right)$ & $\mathrm{m}$ & Coordinate of the drop point \\
\hline
\end{tabular}

The parameters in Tab. 1 can be grouped into 4:

Group 1: Steep glideslope, Shallow glideslope, Horizontal coordinate of starting point of Shallow Glideslope segment can be determined considering the aerodynamics of the demonstrator, flight envelop constrains and engineering experience. 
Group 2: $\mathrm{X}$ coordinate of Starting point of Exponential Decay $\left(\mathrm{X}_{\mathrm{C}}\right)$, Decay rate of the Exponential Flare $(\sigma)$ need iteration of optimization based on the restriction.

Group3: Proportion Factor of Exponential Flare $\mathrm{H}_{\mathrm{DY}}$, Origin of the circular flare arc $\mathrm{K}$, the radius of the arc $\mathrm{R}$, the altitude of the starting point of circular flare $\mathrm{H}_{\mathrm{B}}$, and the touchdown point $\mathrm{A}$ can be determined considering the continuous of trajectory and the restriction of $\mathrm{X}_{\mathrm{C}}$ and $\sigma$.

Group 4: Coordinate of the starting point of Capturing Segment $N$, the radius of the arc $R_{\mathrm{P}}$, the finishing point of arc trajectory $\mathrm{M}$ can be generated by the online self-adaption algorithm based on the current position $(\mathrm{P})$ of the demonstrator at the end of the attitude stabilization segment.

\section{Parameter Selection}

Offline trajectory design needs strict restriction of dropping window, while the online trajectory design reduces its dependence on the pre-designed trajectory, expanding the envelop of initial value constraints, providing the trajectory phase with the maximum robustness, with enhancing the safety and reliability of the demonstrator. So the self-adaption method is used to calculate the capturing segment parameters while the offline method is used in the calculation of parameters in landing segments.

\subsection{Group 1}

a) $\gamma_{1}$

Supposing the dynamic pressure and the path angle in Steep Glideslope stay the same, selected steep glideslope angle maintain the balance of gravity component and the drag. The motion equations of the demonstrator are given by:

$$
\left\{\begin{array}{l}
q S C_{L}=m g \cos \gamma_{1} \\
q S C_{D}=-m g \sin \gamma_{1}
\end{array}\right.
$$

where $q$ is the dynamic pressure, $S$ is the wing span, $C_{L}$ is the lift coefficient, $C_{D}$ is the drag coefficient, $m$ is the demonstrator mass, $g$ the gravity acceleration, and the path angle $\gamma_{1}$ is given by

$$
\tan \gamma_{1}=-\frac{C_{D}}{C_{L}}
$$

Obviously, the path angle $\gamma_{1}$ at any altitude is inverse proportion to the lift-to-drag ratio, meaning that the smaller the lift-to-drag ratio is, the larger $\left|\gamma_{1}\right|$ will be, and the bigger the effection on $\gamma_{1}$ of the lift-to-drag ratio uncertainty will be. Thus, the path angle $\gamma_{1}$ better be picked as small as possible from which can meet the needs of the energy and stability requirements.

b) $\gamma_{2}$

Shallow glideslope angle $\gamma_{2}$ is determined by the velocity and descending rate of touchdown, the relationship of touchdown velocity, descending velocity and shallow glideslope angle $\gamma_{2}$ can be expressed as:

$$
\dot{H}=V \sin \gamma_{2}
$$

The smaller $\left|\gamma_{2}\right|$ is, the smaller descending rate will be, and the easier realized the flare will be. Considering the demonstrator is accelerated in this segment, the parameter $\gamma_{2}$ should be picked according to the descending rate of the landing flare starting velocity of common UAV.

c) Horizontal coordinate of starting point of flare

Horizontal coordinate of starting point of flare $\mathrm{X}_{\mathrm{L}}$, can be determined by the prescribed duration of the landing flare segment. The landing flare segment begins at altitude of 15 meters, and ends after 5 seconds, so $\mathrm{X}_{\mathrm{L}}$ can be given by:

$$
X_{L}=\int_{0}^{5} V(t) \cdot \cos (\gamma(t)) \cdot d t
$$

So, $\mathrm{X}_{\mathrm{L}}$ is determined by the velocity and path angle of the landing flare segment. 


\subsection{Group 2}

The iteration procedure of solving parameters of group 2 is shown in Fig. 2. In every interation step, with the initial altitude $\mathrm{H}_{\mathrm{A}}$ and dynamic pressure $\mathrm{q}_{\mathrm{A}}$ of the landing trajectory already determined by experience, trajectory geometry parameters, including coordinates of Points A, B, C, K and L, is derived from the guessed abscissa of starting point $\left(\mathrm{X}_{\mathrm{C}}\right)$ and decay rate $(\sigma)$ of exponential flare segment. Then a simulation (Barton and Tragesser, 1999) based on the generated trajectory is carried out, and the dynamic pressure in Point $\mathrm{L}$ and the normal overload at point $\mathrm{C}$ is calulated. If the dynamic pressure in Point $\mathrm{L}$ exceeds the constraint, parameter $\sigma$ will be adjusted for the next interation. If not, when the normal overload at point $\mathrm{C}$ exceeds the constraint, parameter $\mathrm{X}_{\mathrm{C}}$ will be adjusted for the next interation. Once the new parameter $\sigma$ or $\mathrm{X}_{\mathrm{C}}$ adjusted, next interation step begins.

The adjustment of $\sigma$ is trying to find a realizable flight trajectory under the constraints of starting point of landing and starting point of flare. The adjustment of $\mathrm{X}_{\mathrm{C}}$ is processed in the whole flight trajectory, with little influence of experience factor of designers. If normal overload is added to the consideration of design, the change of normal overload will be smaller, making the trajectory smoother.

\subsection{Group 3}

After parameters of groups 1 and 2 and the initial altitude of landing segmants are determined, $\mathrm{H}_{\mathrm{DY}}, R, \gamma_{4}$, and the coordinate of points $\mathrm{A}, \mathrm{B}$ and $\mathrm{K}$ can be determined with derivation of geometry relation.

The proportion factor of the exponential function is given by:

$$
H_{D Y}=\frac{X_{L} \cdot \tan \gamma_{2}}{e^{\left(X_{C}-X_{L}\right) / \sigma}}
$$

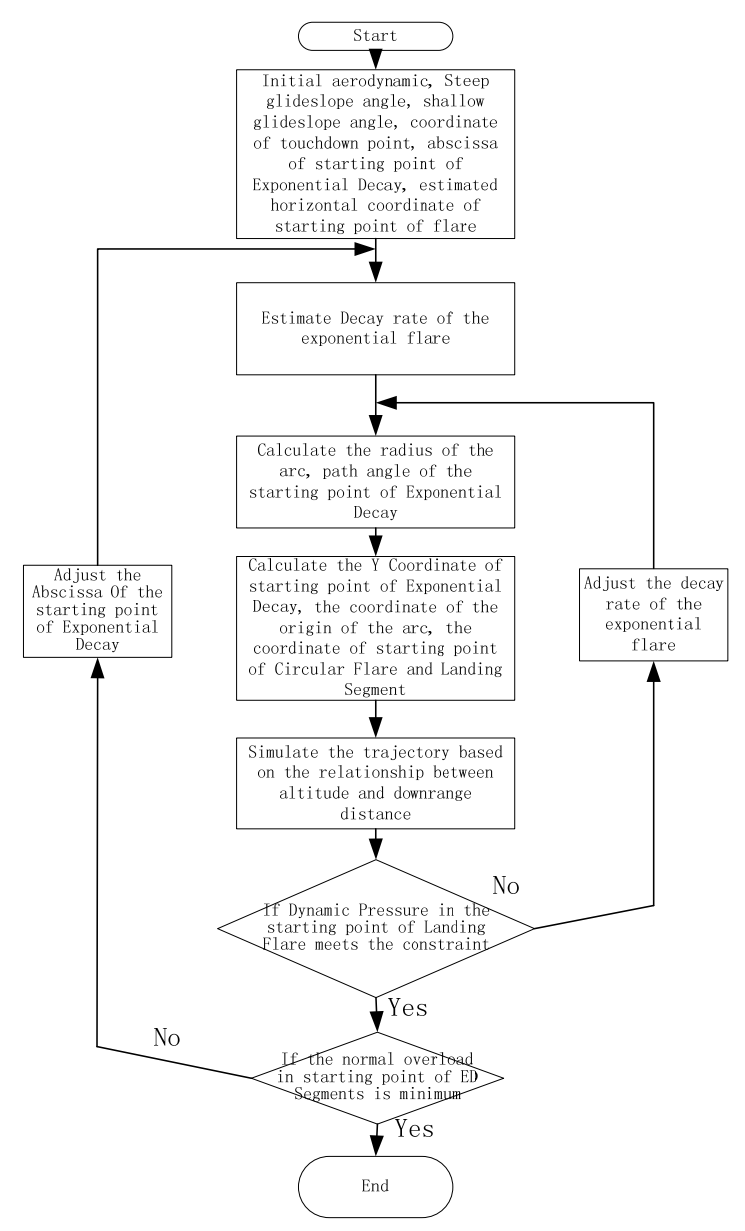

Fig. 2. Flow-chart of solving the altitude phase 
The radius $\mathrm{R}$ is given by:

$$
R=\frac{\sigma^{2}\left(1+\left(\tan \gamma_{2}-\frac{H D Y}{\sigma}\right)^{2}\right)^{\frac{3}{2}}}{H D Y}
$$

The path angle at cross point of the Circular Flare and Exponential Decay $\left(\gamma_{4}\right)$ is given by:

$$
\gamma_{4}=\tan ^{-1}\left(\tan \gamma_{2}-\frac{H_{D Y}}{\sigma}\right)
$$

The coordinate of point $\mathrm{C}$ is given by:

$$
H_{C}=X_{\mathrm{C}} \cdot \tan \gamma_{2}+H_{D Y}
$$

The coordinate of point $\mathrm{B}$ is given by:

$$
\begin{aligned}
& X_{B}=X_{C}+R \cdot\left(\sin \gamma_{1}-\sin \gamma_{4}\right) \\
& H_{B}=H_{C}+R \cdot\left(\cos \gamma_{1}-\cos \gamma_{4}\right)
\end{aligned}
$$

The coordinate of point $\mathrm{K}$ is given by:

$$
\begin{aligned}
& X_{K}=X_{B}-R \cdot \sin \gamma_{1} \\
& H_{K}=H_{B}+R \cdot \cos \gamma_{1}
\end{aligned}
$$

The coordinate of point $\mathrm{A}$ is given by:

$$
X_{A}=X_{B}+\frac{\left(H_{A}-H_{B}\right)}{\tan \gamma_{1}}
$$

\subsection{Group 4}

After the parameters of landing segment determined as above and the altitude of point $\mathrm{M}$ picked based on experience, coordinates of point $\mathrm{M}$ and $\mathrm{N}$ and the radius $\mathrm{R}_{\mathrm{P}}$ can be calculated online with self-adapted algorithm based on demonstrator position, once its orientation is stabilized and the velocity is below constraint in capturing segment.

a) The coordinate of point $\mathrm{M}$ is given by:

$$
X_{M}=X_{A}+\left(H_{M}-H_{A}\right) / \tan \gamma_{1}
$$

b) The radius $R_{P}$ is given by:

$$
R_{P}=\frac{\sqrt{\left(X_{P}-X_{M}\right)^{2}+\left(H_{P}-H_{M}\right)^{2}}}{\gamma_{1}-\tan ^{-1}\left(\frac{H_{P}-H_{M}}{X_{P}-X_{M}}\right)}
$$

c) The coordinate of point $\mathrm{N}$ is given by:

$$
\begin{gathered}
X_{N}=X_{M}-R_{P} \cdot \sin \gamma_{1} \\
H_{N}=H_{M}+R_{P} \cdot \cos \gamma_{1}
\end{gathered}
$$




\section{Gain Scheduling Control}

The gain scheduling control laws of the demonstrator are illustrated in Fig. 3 and Fig. 4. In the attitude stabilization segment, the pitch angle is controlled. While in the capturing segment, the altitude and vertical velocity are controlled, with the altitude command calculated according to equation 1 to 5 and the vertical velocity command generated following changes of the altitude command.

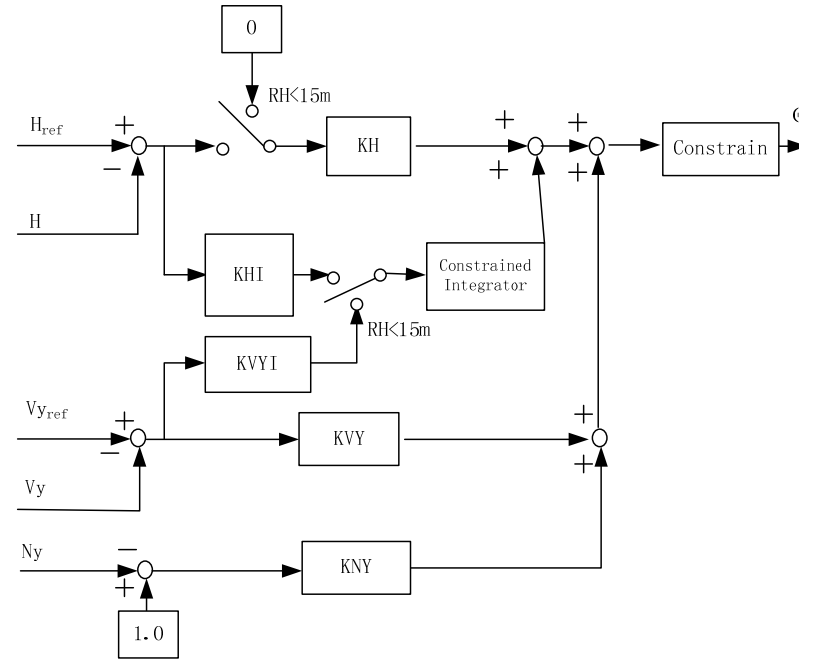

Fig. 3. Vertical Controller

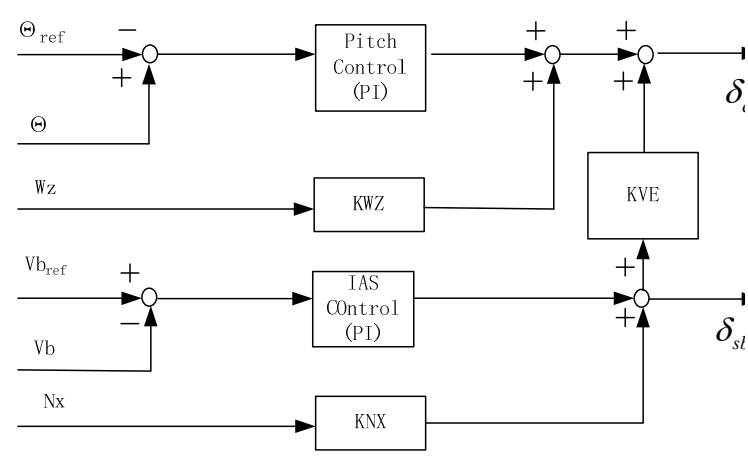

Fig. 4. Pitch Controller

The demonstrator use IAS control (PI) in the velocity control loop, and the command is predetermined offline. The IAS is achieved by using the speedbrake, which will generate an additional moment to the demonstrator. Thus, a cross-link of speedbrake command $\left(\delta_{s b c m d}\right)$ and elevator command $\left(\delta_{\text {ecmd }}\right)$ is introduced to the pitch control law. Besides, the pitch angle rate, the normal overload and the axial overload are also introduced, to enhance the damping of the system.

\section{Simulation and Analysis}

In order to verify the auto-landing solution proposed in this paper, two methods are applied to conducte flight simulations and performance evaluations based on the demonstrator. One is RSS analysis method, which is used to find primary factors that can exacerbate the flight performance and the right direction to improve the solution, by evaluating how different uncertain factors affects the flight performance. The other is Monte Carlo method, in order to evaluate the robustness of the solution by finding out flight performance boundaries with existence of multiple uncertain factors.

Table 2. Flight Performance and the RSS analysis results

\begin{tabular}{llllllll}
\hline Type & Name & Symbol & Unit & Min & Max & $\begin{array}{l}\text { Normal } \\
\text { Value }\end{array}$ & RSS \\
\hline \multirow{4}{*}{ Slapdown } & Down Range departure & XD & $\mathrm{m}$ & 200 & 200 & 0 & 164.81 \\
Performance & Cross Range departure & ZD & $\mathrm{m}$ & -20 & 20 & 0 & 6.53 \\
& Sink Rate & $\mathrm{VyD}$ & $\mathrm{m} / \mathrm{s}$ & -3 & 0 & -0.8 & 0.62 \\
& Ground Velocity & $\mathrm{VD}$ & $\mathrm{m} / \mathrm{s}$ & 0 & 97.22 & 85.79 & 10.12 \\
& Roll Attitude & $\mathrm{GamaD}$ & $\mathrm{deg}$ & -5 & 5 & 0.02 & 3.17 \\
\multirow{2}{*}{$\begin{array}{l}\text { Rollout } \\
\text { Performance }\end{array}$} & $\begin{array}{l}\text { Pitch Attitude } \\
\text { Running Distance }\end{array}$ & ThetaD & $\mathrm{deg}$ & 0 & 10 & 7 & 0.74 \\
& $\begin{array}{l}\text { Maximum Lateral } \\
\text { Taxiing Deviation }\end{array}$ & $\mathrm{ZH}$ & $\mathrm{m}$ & -20 & 20 & 0 & 21.25 \\
\hline
\end{tabular}




\begin{tabular}{llllllll}
\hline & Max Dynamic Pressure & QU & pa & 0 & 13000 & 11175.46 & 803.49 \\
Inflight & Max Normal Overload & NyU & $\mathrm{g}$ & 0 & 2.5 & 1.59 & 0.62 \\
Performance & Max Angle of Attack & AlphaU & deg & -5 & 18 & 8.47 & 4.26 \\
& Max Angle of Sideslip & BetaU & deg & -5 & 5 & 0.46 & 1.82 \\
\hline
\end{tabular}

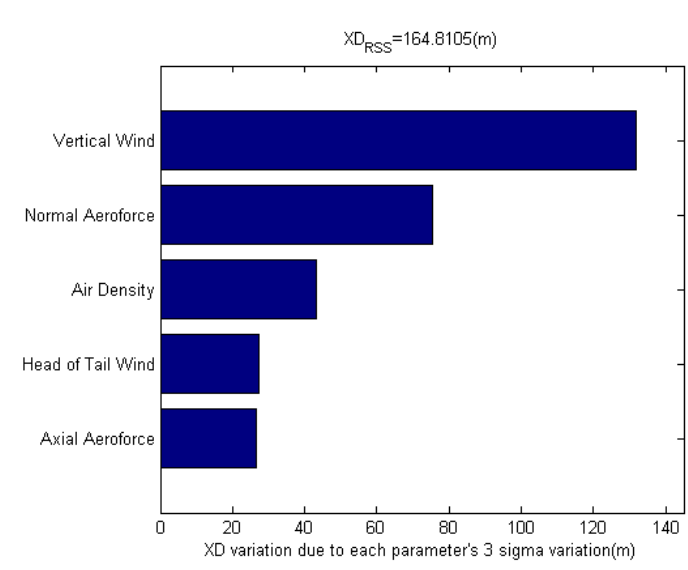

(a)

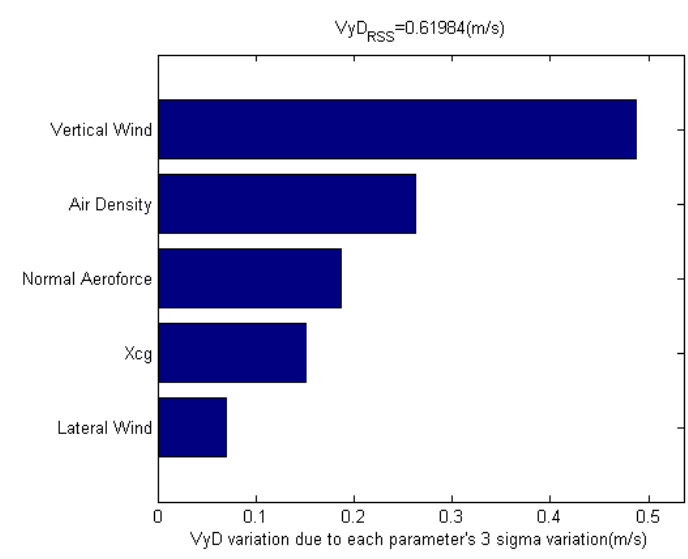

(c)

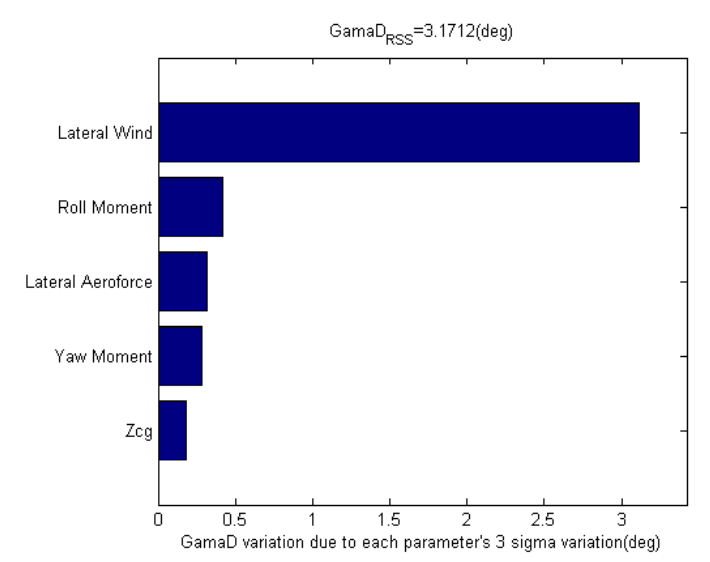

(e)

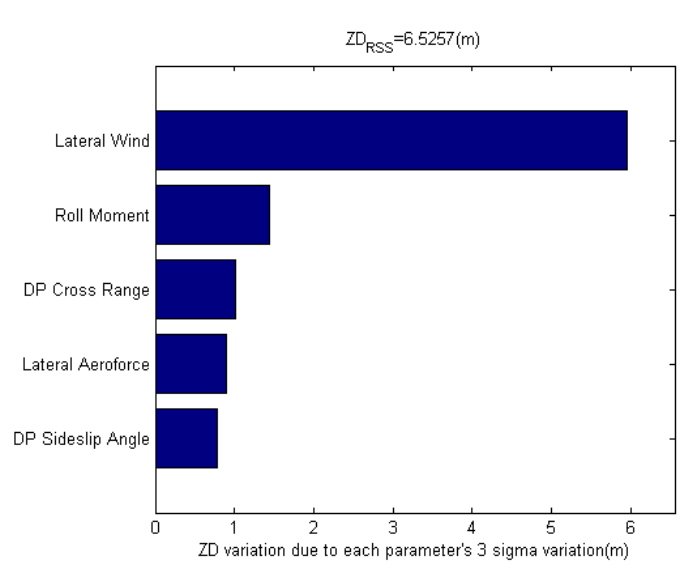

(b)

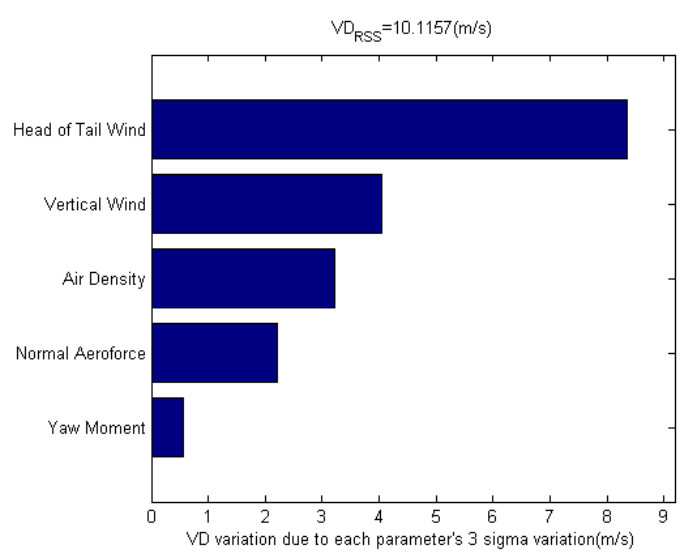

(d)

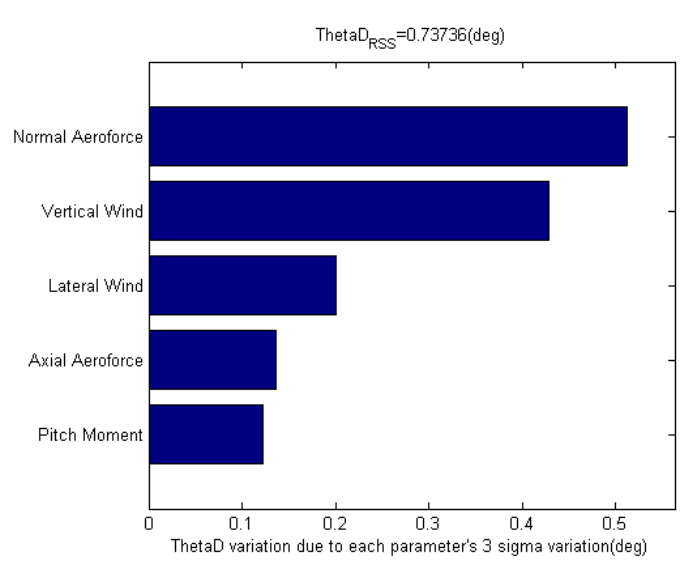

(f) 


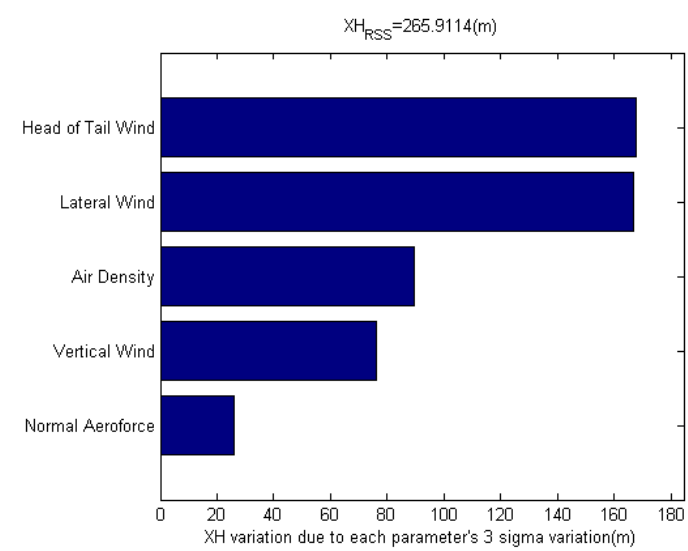

(g)

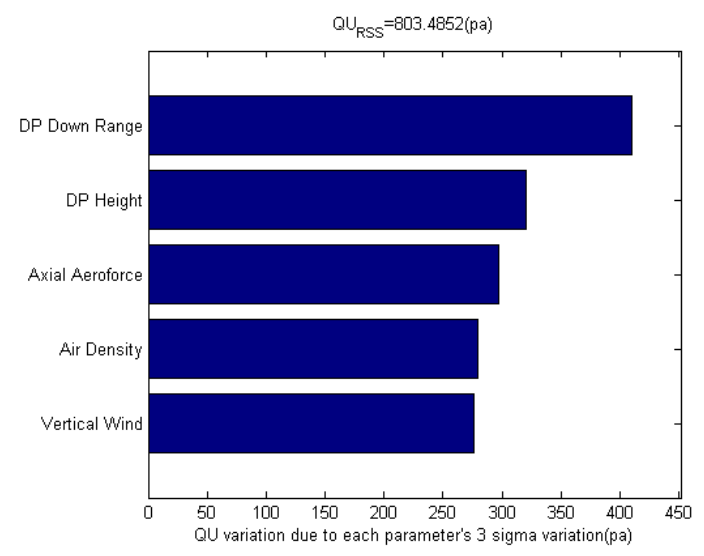

(i)

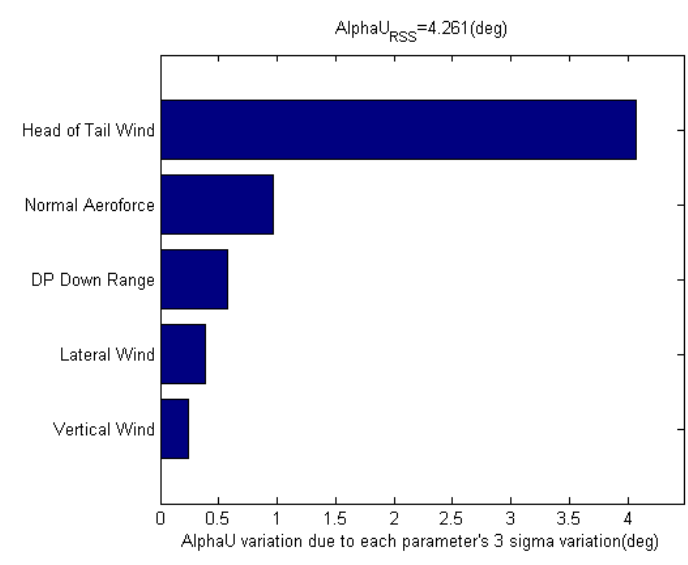

(k)

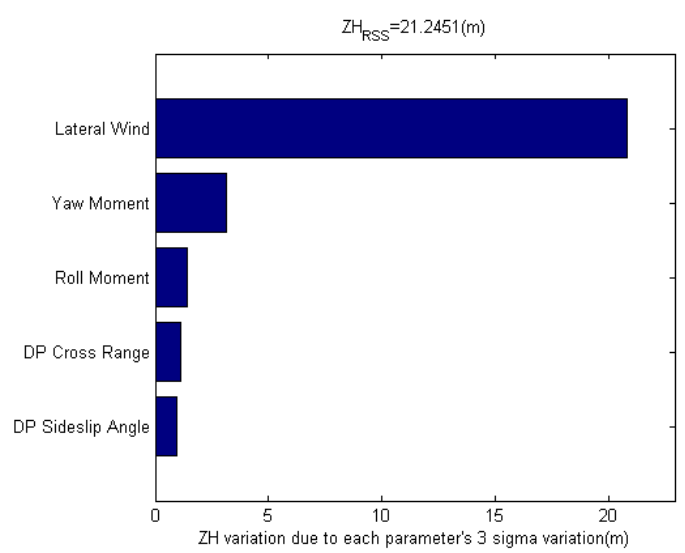

(h)

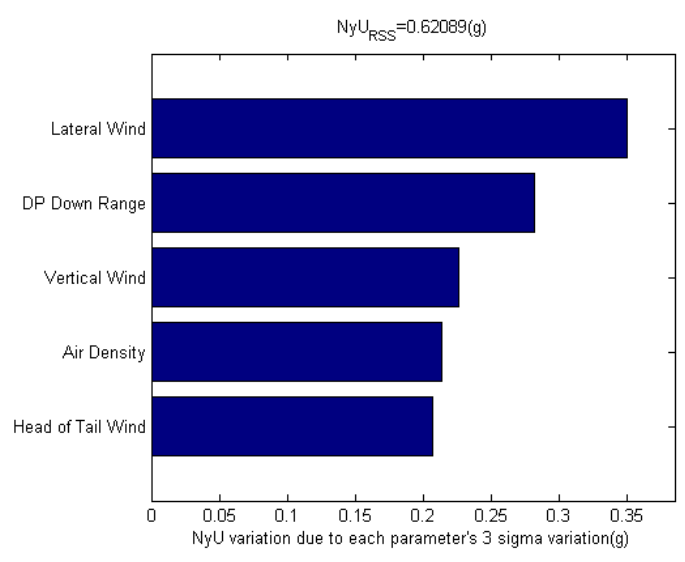

(j)

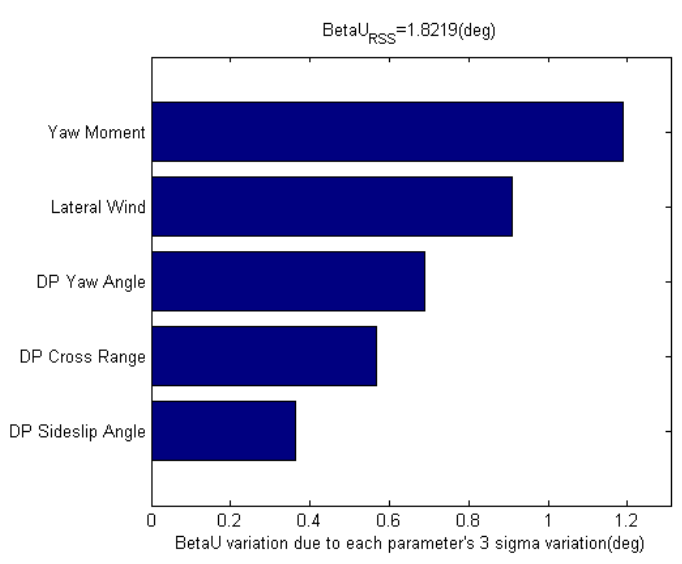

(h)

Fig. 5. Flight performance RSS value and the first five disturbances

In RSS analysis, we primarily researched on the influences of 37 uncertain factors belonging to four categories, which are flight quality characteristics, aero-dynamic parameters, initial drop conditions, and turbulence; we also did our research on 12 flight performance items belonging to three categories, which are touchdown performance, taxiing performance, and in-flight performance. The definitions, units, expected boundary values, standard values and RSS computation results of all these performances are listed in Tab. 2. Fig. 5 shows the RSS analysis results of each performance item as well as the 5 most influential ones. We can see from the results that the velocity at touchdown point and the maximum lateral taxiing deviation are close to or even have exceeded the 
expected performance boundary, the main influential factor of which is the wind field. Besides, the other items of flight performance are acceptable after being disturbed. Therefore, this design still needs to be improved. If not, more rigorous wind field condition of the unpowered drop test should be proposed for the safty of the autolanding; if improved, the anti-wind capability of the aircraft would be increased.

As for the Monte Carlo method, we added sensor measurement errors in 10000 times of simulation. Fig.6 shows the statistical results of the primary flight performance parameters. Star signs are used to mark the expected performance boundary. We can see from the results that the average of the touchdown points, ' $x$ ' marks, is slightly to the right of the standard trajecory. The percentage of the touchdown points that are inside the expected area is $97.2 \%$. The other flight parameters in addition to that can basically satisfy the flight performance boundary. Fig. 7 shows the vertical and lateral flight trajectories, which can apparently converge to the standard trajectory with the existence of multiple uncertain factors. This indicates that the solution has shown an acceptable robustness and can provide enough capability for the demonstrator to land saftly.

Results of the aforementioned two simulations have shown that this autolanding solution is feasible and acceptable. And if the anti-wind capability can be increased, the flight performance would be better improved.

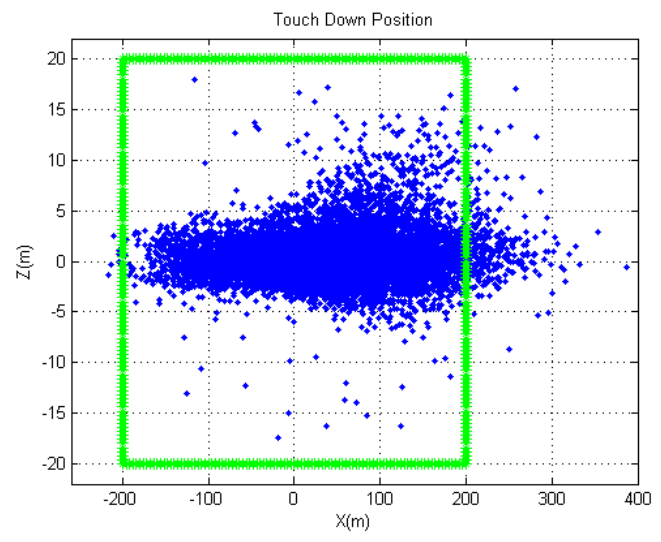

(a)

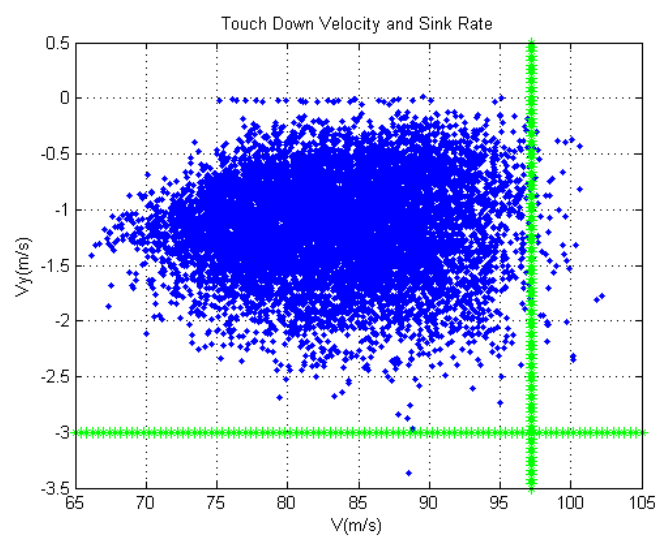

(c)

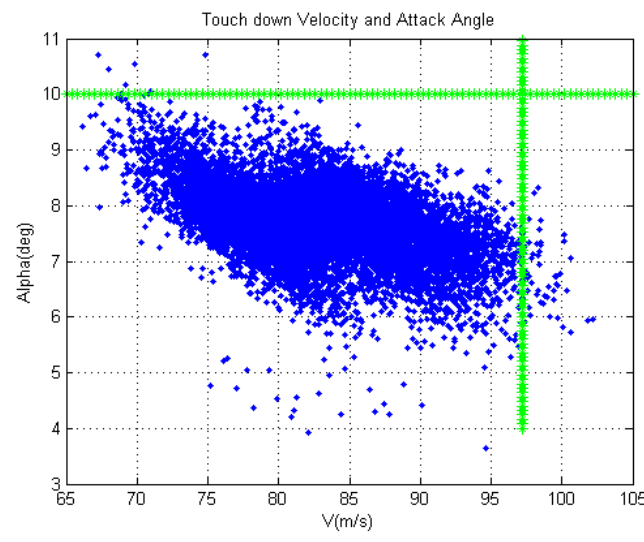

(b)

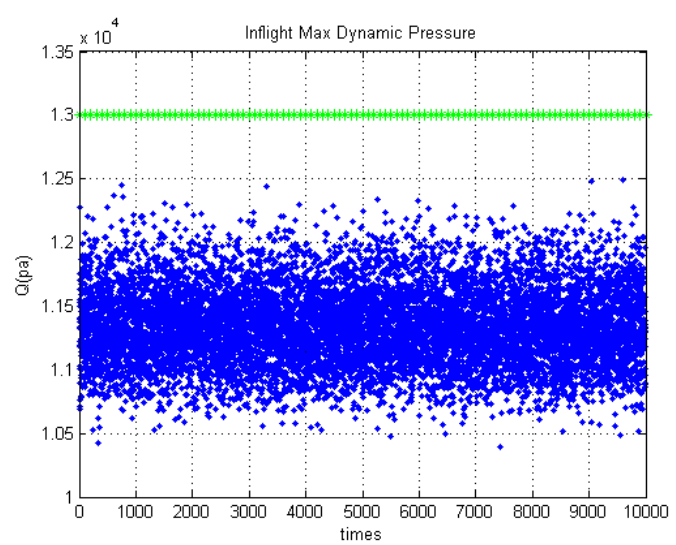

(d)

Fig. 6. Primary flight performance boundaries and Monte Calro simulation results 


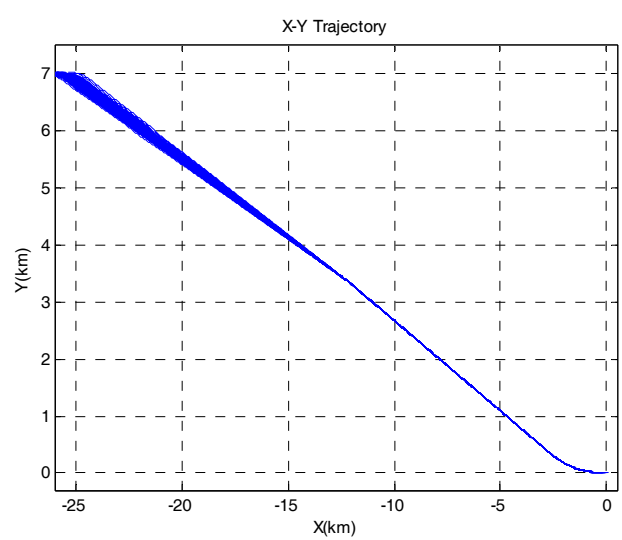

(a)

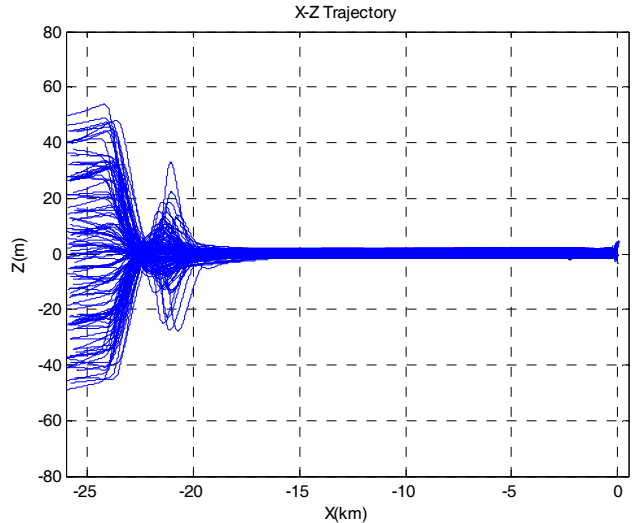

(b)

Fig. 7. Autolanding trajectories results

\section{Conclusions}

To ensure a safe auto-landing of a unpowerred drop test vehicle, a new auto-landing solution is proposed including an on-line trajectory generation method and gain scheduling control laws.

In the generation of autolanding segments, a self-adaped capture segment is considered, an iteration method optimizing the abscissa of starting point of flare segment and decay rate of exponential flare is introduced to reduce effects of human decision, and the end of shallow steep is setted to be the $15 \mathrm{~m}$ high to improve touch down performance, all of which lead to a more robust trajectory generation process. Besides, the classic gain schedulilng control laws are feasible and reliable with simple but useful control mode and logic.

RSS and Monte Carlo Methods are used for simulation analysis and evaluation of the proposed solution in multi-disturbance circumstances. Analysis results prove that the autoland solution is effective and robust to generate a smoothed and energy controllable trajectory for a safe autolanding with multiple uncertain factors.

\section{References}

Ali Heydari, Sivasubramanya Balakrishnan. (2011). Optimal Online Path Planning for Approach and Landing Guidance. AIAA Atmospheric Flight Mechanics Conference, Portland, Oregon. http://dx.doi.org/10.2514/6.2011-6641

Ander R. G. (2001). Onboard Trajectory Generation for Unpowered Landing of Autonomous Reusable Launch Vehicle. MS Thesis, MIT.

C. A. K. (2004). Unpowered approach and landing guidance using trajectory planning. Journal of Guidance, Control, and Dynamics, 27, 967-974. http://dx.doi.org/10.2514/1.7877

C. A. K. (2007). Unpowered Approach and Landing Guidance with Normal Acceleration Limitations. Journal of Guidance, Control, and Dynamics, 30, 882-885. http://dx.doi.org/10.2514/1.28081

Christina T. C. (1998). Design of a robust integrated guidance and control algorithm for the landing of an autonomous reusable launch vehicle. MS Thesis, MIT.

Gregg, H., Barton, S., \& Tragesser, G. (1999). Autolanding Trajectory Design for the X-34. AIAA atmospheric flight conference and exhibit, Portland, Oregon. http://dx.doi.org/10.2514/6.1999-4161

Gust, M. T. (1982). Space shuttle Autoland design. AIAA Guidance and Control Conference San Diego, California. http://dx.doi.org/10.2514/6.1982-1604

Schierman, J. D., \& Ward, D. G. (2001). A reconfigureable guidance approach for reusable launch Vehicle. AIAA Guidance, Navigation, and Control Conference and Exhibit, Montreal, Canada. http://dx.doi.org/10.2514/6.2001-4429

Masaaki Y., Yoshikazu M., \& Hirofumi T. (1999). Simulation analysis of the HOPE-X demonstrator. International Space Planes and Hypersonic Systems and Technologies Conference, Norfolk, VA, U.S.A. http://dx.doi.org/10.2514/6.1999-4875 
Miyazawa, Y., \& Motoda, T. (1999). Longitudinal Landing Control Law for an Autonomous Reentry Vehicle. Journal of guidance, control, and dynamics, 22(6), 791-800. http://dx.doi.org/10.2514/2.4480

Motoda, T., \& Miyazawa, Y. (1998). ALFLEX flight simulation analysis and flight testing, AIAA Aerospace Sciences Meeting and Exhibit, Reno, NV, U.S.A. http://dx.doi.org/10.2514/6.1998-301

Schierman, J. D., Hull, J. R., \& Ward, D. G. (2002). Adaptive guidance with trajectory reshaping for reusable launch vehicle. AIAA Guidance, Navigation, and Control Conference and Exhibit, Montreal, Canada. http://dx.doi.org/10.2514/6.2002-4458

Shen, H. (2004). Autolanding Trajectory Design of Space Shuttle. Flight Dynamics, 22(1), 4-7. (in Chinese).

Sun, C. (2008). Research on Terminal Area Energy Management and Autolanding Technology for Reusable Launch Vehicle. MS Thesis, Nanjing University of Aeronautics and Astronautics (in Chinese).

Sun, C., \& Huang, Y. (2008). Online Trjectory Generation of Shuttle. Missile and Guidance, 28(4), 261-265. (in Chinese).

Toshikazu, M., Yoshikazu, M., Kazutoshi, I., \& Tatsushi, I. (1999). Automatic landing flight experiment flight simulation analysis and flight testing. Journal of Spacecraft and Rockets, 36, 554-560. http://dx.doi.org/ $10.2514 / 3.27199$

\section{Copyrights}

Copyright for this article is retained by the author(s), with first publication rights granted to the journal.

This is an open-access article distributed under the terms and conditions of the Creative Commons Attribution license (http://creativecommons.org/licenses/by/3.0/). 